\title{
CHOIX DES SITES DE FRAIE PAR LA GRANDE ALOSE (ALOSA ALOSA L.) EN MOYENNE GARONNE.
}

\author{
A. BeLAUD (1), A. CARETTE (2), F. CASSOU-LeINS (1), J.J. CASSOU-LEINS (1)
}

(1) INP-ENSAT Environnement aquatique BP107, F-31326 CASTANET-TOLOSAN Cedex. E-mail : belaud@ensat.fr

(2) Association pour la Gestion de la Réserve Naturelle de la frayère d'alose, 22 rue Garonne, F-47520 LE PASSAGE.

\section{RÉSUMÉ}

Dans la période 1995-1999, la forte abondance des migrations de la "grande alose » (Alosa alosa L.) en Garonne a permis de localiser et d'évaluer les frayères dans la partie moyenne du fleuve. Les caractéristiques de 6 sites ont été examinées.

La reproduction a lieu typiquement en zones de plat-courant, avec une profondeur de l'ordre de $2 \mathrm{~m}$ (par moyennes eaux), avec des vitesses de l'ordre de $1 \mathrm{~m} / \mathrm{s}$ sur un substrat de galets propice à la captation des œufs fécondés.

Les sites actifs sont situés sur des tronçons où la pente est localement douce, proches de parcours plus difficiles.

Les profils transversaux sur les aires de ponte conduisent à des vitesses moyennes de l'eau comprises entre 0,8 et $1,5 \mathrm{~m} / \mathrm{s}$, pour les débits habituels de mai (entre 500 et $1000 \mathrm{~m}^{3} / \mathrm{s}$ ). Les profils hors frayères sont traversés par des vitesses supérieures. L'implantation de frayères correspond à un avantage bioénergétique.

L'emplacement des frayères correspond à des segments élargis et/ou plus profonds du fleuve. II est suggéré d'utiliser ces critères pour toute première prospection de nouveaux tronçons de rivières.

A l'échelle du microhabitat et pour le cas de la frayère d'Agen, le positionnement précis des géniteurs traduit l'intérêt des irrégularités des fonds et des décollements du courant des berges bâties ou végétalisées.

Mots-clés : Alosa alosa, reproduction, frayères, habitat, Garonne. 


\section{SPAWNING SITE SELECTION BY ATLANTIC SHAD (ALOSA ALOSA L.) IN THE MIDDLE GARONNE STREAM.}

\section{ABSTRACT}

From 1995 to 1999, the high abundance of migrating fish Alosa alosa in the river Garonne had made it possible to identify and monitor the spawning grounds in the middle part of the river. The characteristics of 6 sites of reproduction were examined.

The reproduction typically takes place in zones of glides, within a depth of about $2 \mathrm{~m}$ (by average water level). It requires an average speed of $1 \mathrm{~m} / \mathrm{s}$ on a substrate of pebbles favourable for the embedding of the fertilized eggs.

The active sites are located on low-sloped sections close to more abrupt slopes.

The transverse profiles on the breeding area resulted in an average water velocity ranging between 0.8 and $1.5 \mathrm{~m} / \mathrm{s}$, for the usual flows of May (ranges between 500 and $1000 \mathrm{~m}^{3} / \mathrm{s}$ ) while beyond these sites, the velocity is usually stronger. The establishment of spawning grounds in low velocity intend to save metabolic energy.

The area of spawning grounds correspond to wider and/or deeper areas of the river. It is suggested that these criterias can be used for the initial assessments of new sites of the river being considered for study.

At the microhabit level, and in the case of the spawning area of Agen, the exact positioning of the breeders refers to the irregularities of the river bed and the starting of the currents from the artificially built or vegetalised banks.

Key-words : Alosa alosa, reproduction, spawning grounds, habitat, Garonne.

\section{INTRODUCTION}

La grande alose (Alosa alosa L.) accomplit une migration génésique en Garonne, qui donne lieu à une pêche d'intérêt (CASTELNAUD et ROCHARD, 1997) et de loisir sur le cours aval du fleuve. La reproduction a lieu habituellement sur quelques segments de rivière délimités. Ce travail s'intéresse aux caractéristiques environnementales de ces sites particuliers, pour discuter le déterminisme de leur choix par les aloses et pour proposer une méthode de prospection des cours d'eau accessibles à ce migrateur.

La population d'aloses en Garonne fut un temps menacée soit directement par la pêche, les barrages, la pollution, etc., soit indirectement par les interventions humaines sur le milieu, destructrices de frayères (CASSOU-LEINS et CASSOU-LEINS, 1981). Au paroxysme des aménagements non ou mal équipés de passes à poissons, en 1986, les pêcheries du système estuarien étaient en baisse (TAVERNY et ELIE, 1988), mais c'est en moyenne Garonne que le stock avait le plus chuté, se réduisant à quelques dizaines de milliers de géniteurs (CASSOU-LEINS et CASSOU-LEINS, 1981). La pérennité de l'espèce reposait alors sur deux principales frayères «traditionnelles » : Agen (aval proche du seuil d'AgenBeauregard) et La Magistère (aval du complexe hydroélectrique de Golfech, mis en service en 1972) (Figure 1). Ces obstacles justifiaient en soi la présence de frayères «forcées ". 


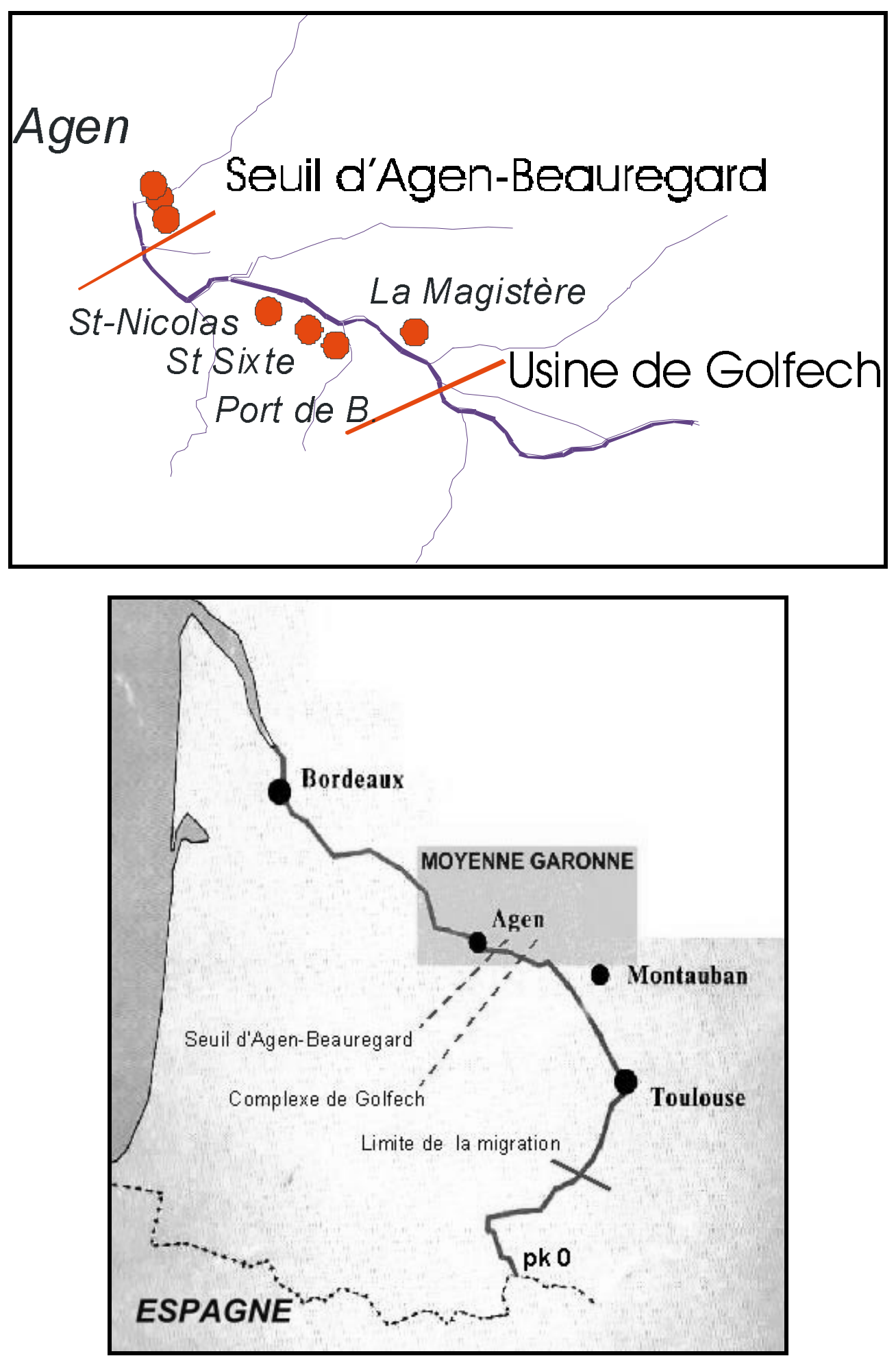

Figure 1

Carte du secteur d'étude.

Figure 1

Map of the site. 
La situation de la grande alose s'est significativement améliorée en moyenne Garonne à partir de 1995 (CHANSEAU et al., comm. pers.) et jusqu'en 1999, avant de marquer un nouveau déclin en 2000. Les améliorations semblaient résulter, d'une part, de la restauration de la circulation des géniteurs et, d'autre part, de la protection de milieux servant de frayères (création de la réserve naturelle de la frayère d'alose à Agen, CASSOU-LEINS et CASSOU-LEINS, 1990). Le recul observé en 2000 montre que ces acquis ne sont pas totalement décisifs.

Les effectifs des années 1995-1999 ont été exceptionnellement favorables à la prospection des frayères d'alose de la moyenne Garonne, sous l'égide de l'Association pour la Gestion de la Réserve Naturelle de la frayère d'alose (PEGUIN, 1997).

Ce travail, mené sur un ensemble de 6 sites régulièrement fréquentés, vise à dégager les caractéristiques environnementales des habitats choisis.

\section{MATÉRIEL ET MÉTHODES}

La ponte des aloses est perceptible depuis les berges par les bruits (et parfois la vue) de remous créés en surface par la nage tournoyante des géniteurs (localement appelée «bull», ROULE, 1924). Chaque action de ponte dure de 4 à 7 secondes. Le phénomène se déroule exclusivement de nuit (entre $0 \mathrm{~h}$ et $5 \mathrm{~h}$ heure locale, soit GMT+2), avec une fréquence maximale entre $2 \mathrm{~h}$ et $3 \mathrm{~h} 30$ (CASSOU-LEINS et CASSOU-LEINS, 1981 ; 1990). La recherche a donc été basée sur des observations, effectuées soit depuis des d'embarcations dérivantes soit depuis des postes d'écoute en berges. Les frayères connues des riverains ont été privilégiées puis l'ensemble du tronçon Golfech-Agen a été prospecté en exploitant tous les accès.

Une fois répertoriées, les frayères ont été surveillées et évaluées directement par la présence des observateurs postés ou indirectement par utilisation de systèmes d'enregistrements sonores (TROULHET, 1992). L'audition directe étant plus performante, la mobilisation d'observateurs est essentielle et l'appareillage des sites ne constitue qu'un appoint.

Les frayères «traditionnelles » d'Agen et de La Magistère font l'objet d'un suivi nuit par nuit depuis 1981 et 1989 (respectivement). Ces sites sont équipés depuis 1989 d'appareils d'enregistrement fonctionnant de mai à juillet, contrôlés sur place au moins 2 fois par semaine. Le fonctionnement de ces frayères a servi de référence pour l'investigation de nouveaux sites.

Les nouveaux sites repérés en 1997 (PEGUIN, 1997) ont été périodiquement visités pour des comptages de « bulls » sur place ou pour poser des enregistreurs autonomes. Le dépouillement de ces écoutes partielles est complété, pour les nuits manquantes, par interpolation guidée par les tendances mesurées aux frayères de référence.

Ces activités de frayères sont d'un côté sous-estimées par ignorance des signaux sonores trop lointains ou par recouvrement temporel de plusieurs «bulls ». A l'opposé, il est possible que des «bulls » ne donnent pas lieu à la ponte (particulièrement en début et en fin de saison, où ces phénomènes sont anormalement brefs). Une estimation grossière de la fréquentation annuelle de chaque site peut se faire sur la base de 5 à 7 « bulls " par femelle selon CASSOU-LEINS et CASSOU-LEINS (1981), ou en 10 à 12 « bulls » par femelle, selon DARTIGUELONGUE et FATIN, 1995.

Des données environnementales ont été extraites de documents existants. La pente du fleuve a été retracée à partir de la compilation de données effectuée par LAMBERT (1989). Les largeurs ont été relevées sur des cartes et des photographies aériennes 
verticales (campagne d'août 1983). Enfin des profils récents ont été mis à disposition par la DDE (Tarn et Garonne et Lot et Garonne), afin d'évaluer les surfaces mouillées pour diverses cotes (correspondant à différents débits).

L'exploitation des profils en travers a consisté à tracer les lignes de niveau pour diverses valeurs de débit. Ces niveaux ont été déterminés soit directement par une station de jaugeage locale soit, à défaut, par interpolation entre deux stations encadrant le profil considéré. Les sections mouillées correspondantes ont été mesurées par planimétrie. Les vitesses moyennes pour chaque débit ont été calculées par division de la valeur de débit par la section mouillée correspondante. Pour chaque profil, un ensemble de points ainsi obtenus génère une caractéristique vitesse $=\mathrm{f}$ (débit).

Les données hydrologiques et climatiques ont été aimablement fournies par divers services (DDE Lot et Garonne, Météo-France à Agen).

L'approche environnementale a été complétée par des mesures spécifiques au niveau de la réserve d'Agen : vitesses en surface par un courantomètre MARSH

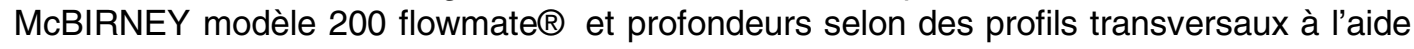
d'un échosondeur installé sur une embarcation.

\section{RÉSULTATS}

Les frayères de la moyenne Garonne se situent sur 4 lieux reportés dans la carte de la Figure 1. Le Tableau I précise les points kilométriques (par rapport au point 0 marquant l'entrée de la Garonne en territoire français). Certains de ces lieux ne contiennent qu'un seul segment fréquenté par les géniteurs d'alose ; d'autres englobent plusieurs segments séparés. La réserve naturelle de la frayère d'aloses, à Agen, va du pk 291,13 au pk 193,20 et comprend 3 segments de ponte d'aloses désignés F1, F2 et F3. Le site Port de Bonneau / St-Sixte est tantôt unique, tantôt fragmenté en 2 ou 3 segments distincts. Le Tableau I récapitule les principales données biologiques et environnementales de chacune des frayères analysées.

\section{Tableau I}

Liste, position et fonctionnement (nombre estimé de pontes) des frayères d'alose de la moyenne Garonne.

Table I

List, longitudinal position and annual evaluation (estimated number of spawns) of shad spawning areas in the middle Garonne river.

\begin{tabular}{|c|c|c|c|c|c|c|}
\hline Frayère & position & \multicolumn{5}{|c|}{ Activité } \\
\hline & pk & 1996 & 1997 & 1998 & 1999 & 2000 \\
\hline Agen F1 & $290,8-291,6$ & & & 0 & 4284 & $<1000$ \\
Agen F2 & $291,8-296,4$ & & & 12000 & 25924 & 13628 \\
Agen F3 & $292,8-293,1$ & & & 22000 & 42172 & 19888 \\
total AGEN & & 3000 & 16120 & 34000 & 72380 & 34000 \\
& & & & & & \\
St-Nicolas de la B. & $275,0-275,6$ & $?$ & $?$ & 350000 & 10500 & 12885 \\
St-Sixte + Port de Bonneau & $270,6-271,9$ & $?$ & $?$ & 300000 & 40700 & 15397 \\
La Magistère & $266,6-269,3$ & 270000 & 169000 & 350000 & 211000 & 27760 \\
total année & & & & 1000000 & 335000 & 90000 \\
\hline
\end{tabular}


Le profil en long de la Garonne depuis l'estuaire et jusqu'à la zone étudiée est représenté dans la Figure 2. La photographie aérienne de la Figure 3 illustre l'intérêt de ces documents pour délimiter les zones de plats et de radiers, relever les largeurs et repérer les segments élargis et autres singularités.

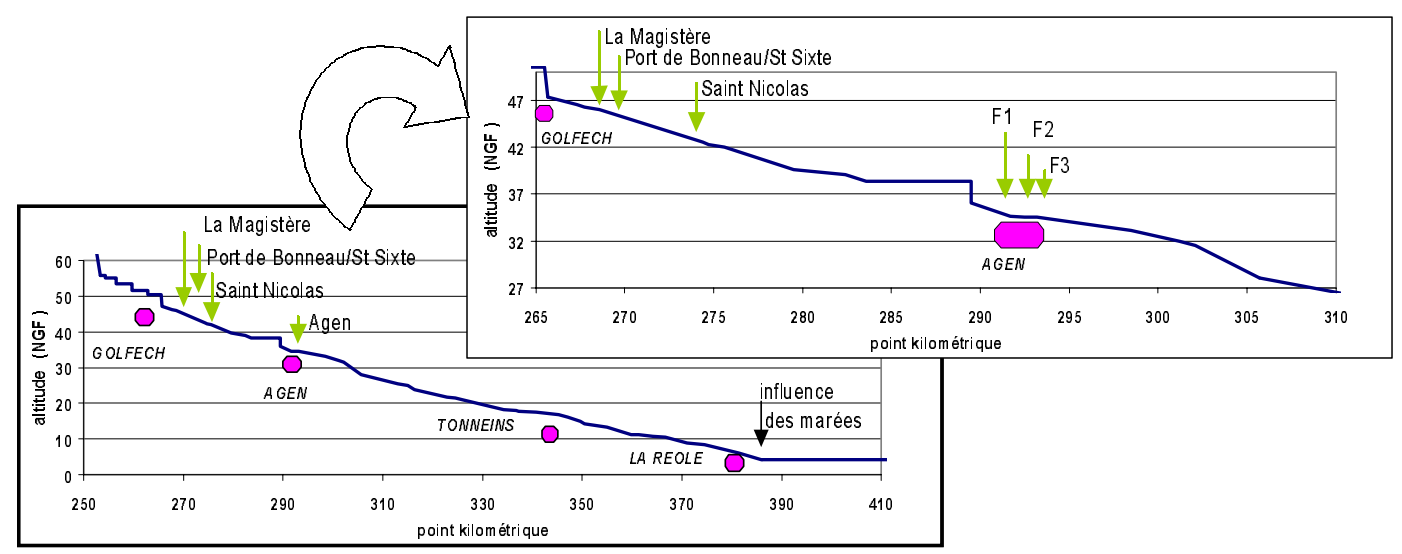

\section{Figure 2}

Profil en long de la Garonne entre l'estuaire et Golfech.

\section{Figure 2}

Longitudinal profile of the middle and low Garonne river.

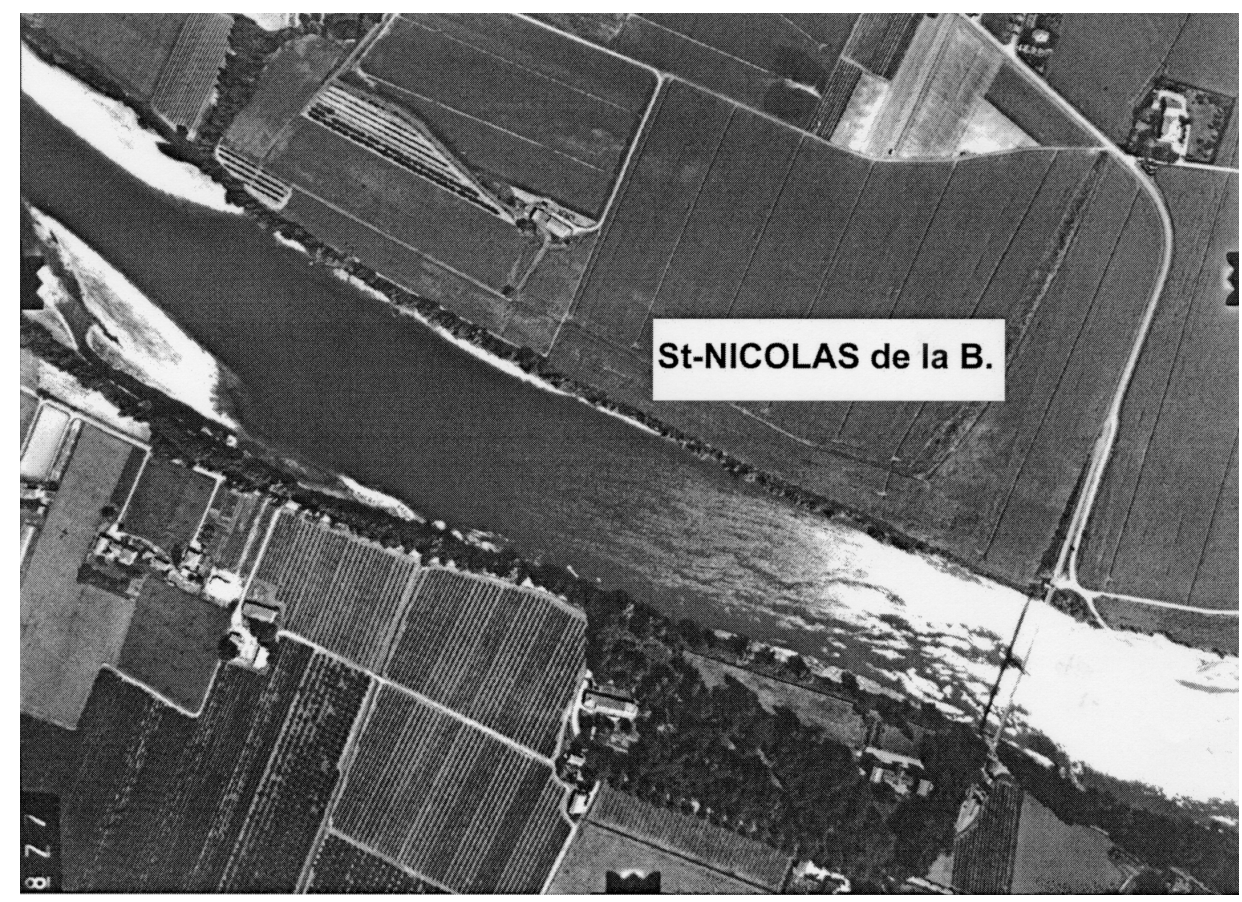

\section{Figure 3}

Photographie aérienne d'un tronçon de Garonne incluant un site de frayère.

\section{Figure 3}

Aerial photograph of the river, above one of the spawning sites. 
La Figure 4 montre un exemple de profil et de courbe (vitesse moyenne vs débit) correspondant à un site de fraie. La Figure 5 compare les vitesses moyennes ainsi calculées sur les profils disponibles dans ou en dehors des frayères.
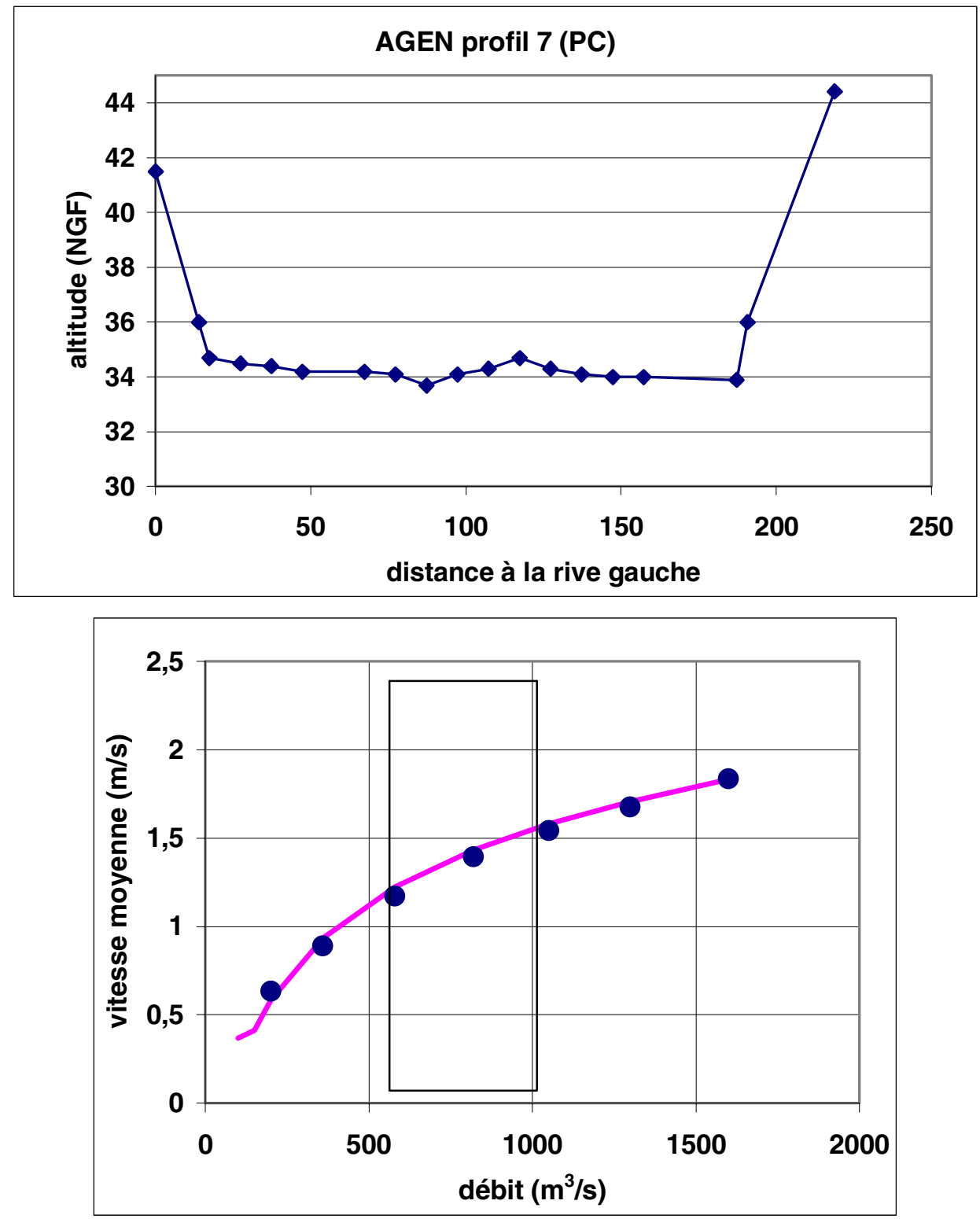

\section{Figure 4}

Exemple de profil transversal et des valeurs de vitesse moyenne calculée pour diverses valeurs de débit du fleuve. La partie encadrée correspond aux débits habituels en mai, lorsque les aloses s'installent en Garonne.

\section{Figure 4}

Example of cross-section and mean water velocity calculated for various discharge, especially in the range $500-1000 \mathrm{~m}^{3} / \mathrm{s}$ encountered in May. 

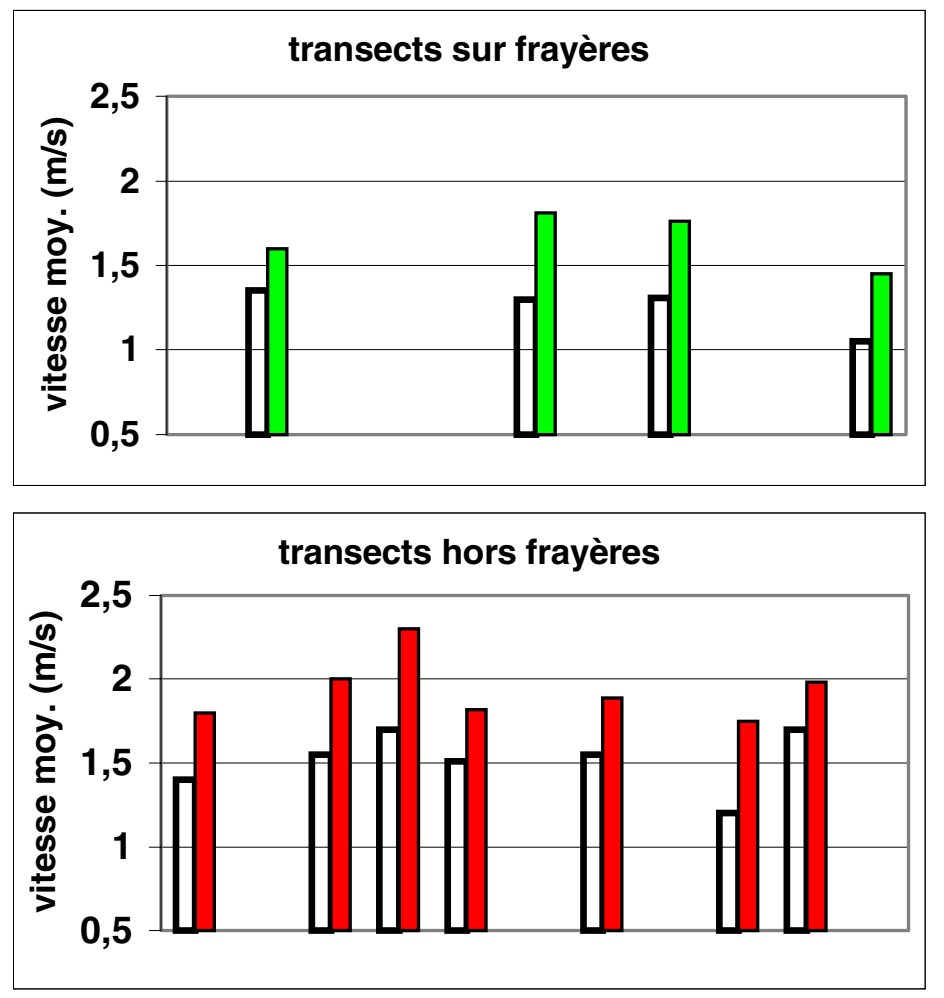

\section{Figure 5}

Comparaison des gammes de vitesses moyennes en mai, calculées sur divers profils, correspondant ou non à des frayères d'alose (Barres vides : débit de $500 \mathrm{~m}^{3} / \mathrm{s}$; barres pleines : débit de $1000 \mathrm{~m}^{3} / \mathrm{s}$ ).

\section{Figure 5}

Comparison of the usual water velocity range in May, at different transversal profiles either on spawning sites (up) or outside (down) (open bars : discharge $500 \mathrm{~m}^{3} / \mathrm{s}$; solid bars : $\left.1000 \mathrm{~m}^{3} / \mathrm{s}\right)$.

\section{DISCUSSION}

Les mécanismes habituellement évoqués pour le choix des sites de reproduction des poissons migrateurs combinent les données locales d'habitat et des facteurs biotiques comme le phénomène de retour à la rivière (voire à la frayère) natale. Bien que ceci n'ait pas été formellement démontré, il est probable que la grande alose (Alosa alosa L.) reste inféodée à son bassin d'origine (TAVERNY, 1991). Mais, contrairement aux saumons où les juvéniles marquent une ou plusieurs années les zones de reproduction (SAGLIO, 1993), les frayères d'aloses sont désertées par l'espèce en automne (CASSOU-LEINS et CASSOU-LEINS, 1981). L'hypothèse d'une mémorisation des sites par des géniteurs multi-reproducteurs est peu probable pour la grande alose, qui meurt en quasi-totalité à la première migration. Préalablement à la recherche d'éventuels mécanismes d'imprégnation sensitive et de retour à la frayère natale, il convenait d'examiner les particularités de l'habitat physique des sites utilisés dans les portions de rivières accessibles. L'activation systématique, chaque année, des mêmes sites, attire l'attention sur des paramètres environnementaux à faible variabilité interannuelle. 
En Garonne, la limite d'accessibilité pour les aloses a été, dès 1991, repoussée à $40 \mathrm{~km}$ en amont de Toulouse (barrage de Carbonne) et les présents résultats s'inscrivent dans un équilibre fondé sur une aire de migration proche de ses limites naturelles et ne comprenant que 3 difficultés partielles (seuil d'Agen-Beauregard, complexe hydroélectrique aménagé de Golfech et barrages équipés de Toulouse).

A une échelle globale, le premier critère d'existence de frayères est celui de la difficulté d'accès, aussi bien au niveau des ouvrages artificiels (dans le cas présent AgenBeauregard et Golfech) que de celui des portions libres où la pente est localement accentuée. Le profil en long de la Garonne à l'étiage indique des pentes faibles et régulières de moins de $30 \mathrm{~cm}$ par kilomètre entre la zone d'influence des marées et Sérignac sur Garonne/St Hilaire de Lusignan (pk 305). Ici, la pente s'accentue transitoirement à $70 \mathrm{~cm}$ par kilomètre avant de retrouver l'ordre de grandeur des tronçons aval. La frayère d'Agen est un segment de très faible pente $(7 \mathrm{~cm}$ par kilomètre à l'étiage entre le pont de Pierre et le pont Canal), encadré par des pentes de l'ordre de $20 \mathrm{~cm}$ par kilomètre. Ainsi les aloses trouvent à Agen un plateau de repos sur le trajet migratoire. L'identification de ces zones à profil propice nécessite des repères d'altitude suffisamment resserrés.

A l'échelle spatiale de la frayère, les sites intermédiaires (PEGUIN, 1997 ; BARROIS, 1997), placés sur des tronçons libres, correspondent indiscutablement aux préférences d'habitat des géniteurs d'aloses. Les sites traditionnels de fraie d'Agen et de La Magistère étant restés fonctionnels après restauration de la circulation des poissons (CASSOU-LEINS et CARETTE, 1988 ; BELAUD et CARETTE, 1998 ; 1999), peuvent aussi être considérés comme intéressants en soi et non comme des sites « forcés ».

Les premières observations de CASSOU-LEINS et CASSOU-LEINS (1981), concernant les frayères « traditionnelles » d'Agen et La Magistère, avaient montré que le site de reproduction des aloses s'organise au niveau d'une mouille, suivie immédiatement à l'aval par une zone de radier. La mouille correspond à la zone de fraie proprement dite, et le radier à la zone d'incubation des œufs. II se confirme pour l'ensemble des 6 sites de la moyenne Garonne que la fraie des aloses se produit en faciès typiques de plat-courant, avec une profondeur de l'ordre de $2 \mathrm{~m}$, avec des vitesses de l'ordre de $1 \mathrm{~m} / \mathrm{s}$, où le substrat de galets est propice à la captation des œufs fécondés en surface. Les radiers sont exclus.

Le choix de faciès plats en secteurs de plateaux peut résulter d'une stratégie d'épargne énergétique, souvent évoquée dans le positionnement des poissons en milieux courants. Pour l'alose américaine ( $A$. sapidisissima), les études enzymatiques de la mobilisation des réserves énergétiques montrent des variations selon le niveau atteint dans la rivière et selon les conditions hydrologiques annuelles (LEONARD et McCORMICK, 1999). Le coût énergétique de la migration a été évalué entre 42 et 24 kilojoules par kilogramme de géniteur et par kilomètre parcouru dans la rivière Connecticut (GLEBE et LEGGETT, 1981).

Les profils transversaux au niveau de plats-courants de la moyenne Garonne indiquent que la vitesse moyenne de l'eau sur les profils des sites de frayère est, pour les débits habituellement rencontrés en mai (entre 500 et $1000 \mathrm{~m}^{3} / \mathrm{s}$ ), comprise entre 0,8 et $1,5 \mathrm{~m} / \mathrm{s}$. L'emplacement des frayères correspond ainsi à des segments élargis et/ou plus profonds du fleuve. Les profils hors frayères sont traversés par des vitesses supérieures, et ceci accrédite l'hypothèse de la contrainte énergétique. On peut remarquer que les vitesses maximales tolérées par la grande alose sont nettement supérieures à celles rencontrées par l'alose (Alosa sapidissima) de la rivière Delaware. Dans ce cas, ROSS et al., 1993 décrivent les sites de fraie comme des milieux courants, à vitesses comprises entre 0 et $0,7 \mathrm{~m} / \mathrm{s}$. 
A l'échelle du microhabitat et pour le cas de la frayère d'Agen, deux profils, situés sur des zones inactives de la frayère, se caractérisent par une forme de grande cuvette peu diversifiée et sans hauts-fonds. Un profil sur la zone F1 est comparable aux précédents mais a été le siège, en 1999, de pontes en rive gauche, à la faveur d'un léger élargissement du fleuve, créant un décrochement du courant. Ainsi la zone F1 de la frayère serait un site intéressant essentiellement par fortes eaux. Enfin, 4 profils sur les zones F2 et F3 présentent une alternance de hauts fonds et de sillons profonds qui constituent à l'évidence des abris hydrauliques. Les postes de pêche à la ligne, indiquant les concentrations de poissons, correspondent bien à ces singularités hydrauliques.

Bien que n'excluant pas l'existence d'un déterminisme biotique (justifiant des programmes de recherche spécifiques), l'ensemble de ces éléments soutient le rôle de l'environnement physique.

Pour rechercher des sites de fraie potentiels le long d'une rivière fréquentée par l'alose, le premier tri des sites dans les tronçons de rivières ouverts aux aloses peut se faire selon les faciès d'écoulement, en retenant les plat-courants précédant les radiers. Le deuxième critère consiste à privilégier l'aval proche des difficultés de progression migratoire, artificielles ou naturelles (dont les accentuations de pente au dessus de $0.3 \%$ ), le premier plat situé en aval d'une difficulté étant probablement fréquenté par les aloses. La troisième sélection, sur cartes ou vues aériennes, repère les tronçons élargis de la rivière, donnant lieu à une atténuation particulière des vitesses par temps de crues. Pour affiner cette sélection, les vitesses moyennes en situation hydrologique printanière peuvent être déterminées sur les segments potentiels en utilisant les profils disponibles, pour retenir les zones où cette vitesse n'excède pas $1,5 \mathrm{~m} / \mathrm{s}$. L'irrégularité des fonds apparaissant sur les profils pourra être considérée comme élément favorable supplémentaire.

En appliquant cette démarche de caractérisation des frayères d'aloses à l'ensemble de la moyenne Garonne, on détermine, hors des frayères décrites plus haut, 6 sites supplémentaires (dont 4 en aval d'Agen et 2 intercalés entre des frayères de la moyenne Garonne), qui ne sont pas actuellement fréquentés par les aloses. La méthode proposée semble donc excessive et pourra encore être affinée avec les observations futures en Garonne et autres cours d'eau concernés par la grande alose. Pour l'instant, on peut considérer que ces sites potentiels mais non utilisés constituent a priori une réserve d'espace propice.

Toutefois, l'hypothèse d'un ajustement du nombre de sites fréquentés à l'affluence des géniteurs d'alose ne se vérifie pas car la liste des sites actifs est inchangée en 2000 (faible migration) comparativement à 1998 (nombre de pontes maximal enregistré en moyenne Garonne). II n'apparaît aucune évidence ni de seuil d'affluence nécessaire à la création d'une frayère, ni de phénomène de saturation d'une frayère donnée. Les sites régulièrement fréquentés sont à considérer comme en soi propices à la ponte des aloses, indépendamment des variations hydroclimatiques interannuelles.

La variabilité annuelle porte sur la proportion de géniteurs d'aloses qui s'arrête sur chaque site (Tableau I et données antérieures reprises par BELLARIVA, 1998 et MARTINVANDEMBULKE, 1999). Ainsi, en 1999, caractérisé par de forts débits en mai, les géniteurs d'aloses se seraient arrêtés plus en aval qu'en 1998. Cette répartition semble obéir à un compromis bioénergétique entre, d'une part, une quantité limitée de réserves métaboliques et, d'autre part, un comportement d'avancée maximale dans le fleuve. Le profil de la Garonne et l'hydraulique en période migratoire graduent la difficulté de la migration et provoquent un étagement variable de la population, mais exclusivement sur des sites propices à la reproduction. 


\section{BIBLIOGRAPHIE}

BARROIS J.P., 1997. Reproduction de la Grande alose (Alosa alosa L.) entre Agen et Golfech. Mémoire IUT de Tours, 60 p. + annexes.

BELAUD A., CARETTE A., 1998. Suivi 1998 de la qualité des milieux et de la reproduction des aloses sur le tronçon Agen-Golfech de la Garonne. Rapport Assoc. De la Réserve naturelle de la Frayère d'Alose, $36 \mathrm{p}$.

BELAUD A., CARETTE A., 1999. Suivi 1999 de la qualité des milieux et de la reproduction des aloses à Agen et en moyenne. Rapport Assoc. De la Réserve naturelle de la Frayère d'Alose, $44 \mathrm{p}+$ annexes.

BELLARIVA J.L., 1998. Contribution à l'étude du déroulement de la migration et de la reproduction de la grande alose (Alosa alosa L.) en Garonne. Thèse Doct., Institut National Polytechnique, Toulouse, $103 \mathrm{p}$.

CASSOU-LEINS J.J., CARETTE A., 1988. Réserve naturelle de la frayère d'alose d'Agen : synthèse. Rapport Assoc. De la Réserve naturelle de la Frayère d'Alose, $30 \mathrm{p}$.

CASSOU-LEINS F., CASSOU-LEINS J.J., 1981. Recherches sur la biologie et l'halieutique des migrateurs de la Garonne et principalement de l'Alose (Alosa alosa L.). Thèse 3è cycle, Institut National Polytechnique, Toulouse, $382 \mathrm{p}$.

CASSOU-LEINS F., CASSOU-LEINS J.J., 1990. Réserve naturelle de la frayère d'alose. Synthèse quinquennale et observations complémentaires sur l'Alose, sa reproduction, les larves, les alosons, le milieu. Rapport ENSA Toulouse, $49 \mathrm{p}$.

CASTELNAUD G., ROCHARD E., 1997. Surveillance halieutique de l'estuaire de la Gironde : suivi statistique 1995 - étude de la faune circulante 1996. Rapport CEMAGREF Bordeaux/EDF, $152 \mathrm{p}$.

DARTIGUELONGUE J., FATIN D., 1995. Etude préliminaire de la reproduction des aloses en 1995 entre Tulières et Mauzac sur la Dordogne. Rapport SCEA pour EDF Energie Aquitaine D.T.E., 19 pages + planches, graphiques et annexes.

GLEBE B.D., LEGGETT W.C., 1981. Temporal, intrapopulation differences in energy allocation and use by American shad (Alosa sapidissima) during the spawning migration. Can. J. Fish. Aquat. Sci., 38 (7), 795-805.

LAMBERT R., 1989. Schéma de protection contre les eaux de la Garonne. Atlas hydraulique de la Garonne. Syndicat Mixte d'Etude et de Programmation pour l'aménagement de la Garonne, Toulouse, $82 \mathrm{p}$.

LEONARD J.B.K., McCORMICK S.D., 1999. The effect of migration distance and timing on metabolic enzyme activity in an anadromous clupeid, the American shad (Alosa sapidissima). Fish Physiol. Biochem., 20 (2), 163-179.

MARTIN-VANDEMBULKE D., 1999. Dynamique de population de la grande alose (Alosa alosa L. 1758) dans le bassin versant Gironde-Garonne-Dordogne (France) : analyse et prévision par modélisation. Thèse Doct., Institut National Polytechnique, Toulouse, $115 \mathrm{p}$.

PÉGUIN F., 1997. Caractérisation des sites de frayères de la grande alose entre Agen et la centrale de Golfech. Mémoire IUT de Tours, $63 \mathrm{p}+$ annexes.

ROSS R.M., BENNETT R.M., BACKMAN T.H.W., 1993. Habitat use by spawning adult, eggs and larval American shad in the Delaware River. Rivers, 4 (3), 227-238.

ROULE L., 1924. Les aloses des eaux douces de France. Bull. Soc. Zool. Fr., 49, 265266.

SAGLIO P., 1993. Le retour au site de fraie ou "homing": mécanismes chimiosensoriels. In: Le saumon atlantique, GUEGUEN J. et PROUZET P. (Eds.), Publication IFREMER, 87-100.

TAVERNY C., 1991. Contribution à la connaissance de la dynamique des populations d'aloses (Alosa alosa et Alosa fallax) dans le système fluvio-estuarien de la Gironde: Pêche, Biologie, Ecologie. Etude particulière da la dévalaison et de l'impact des activités humaines. Thèse Doct. Université de Bordeaux/CEMAGERF div. ALA Bordeaux, $451 p+$ annexes. 
TAVERNY C., ELIE P., 1988. Mortalités engendrées par l'industrie de la Pêche. Le cas des juvéniles d'Alosa alosa et d'Alosa fallax dans l'estuaire de la Gironde en 1986. Rapport CEMAGREF Bordeaux, $51 \mathrm{p}$.

TROULHET J.F., 1992. Etude et réalisation d'un dispositif de reconnaissance automatique de signature acoustique. Thèse Doct. Institut National Polytechnique, Toulouse, $113 \mathrm{p}$. 